\title{
REVISIONES
}

\section{TBL - Aprendizaje Basado en Equipos}

\author{
TBL - Team-Based Learning \\ TBL - Aprendizagem Baseada em Equipes
}

Daniel Moraga, ${ }^{a}$ Jeannette Soto

Facultad de Ciencias y Educación, Universidad Iberoamericana de Ciencias y Tecnología, Chile

Correo electrónico: daniel.moraga@uibero.cl

\begin{abstract}
RESUMEN
El presente artículo describe las etapas de la estrategia instruccional de Aprendizaje Basado en Equipos o TBL (del inglés "Team-Based Learning") y una visión general de los resultados de su aplicación en el aula. La evidencia indica que TBL es una estrategia instruccional práctica, económica y efectiva que genera aprendizaje activo en equipos de estudiantes, incrementa la motivación estudiantil, facilita el logro de resultados de aprendizajes, y tiende a mejorar indicadores académicos que impactan en la acreditación de las carreras.
\end{abstract}

Palabras clave: aprendizaje activo y colaborativo, aprendizaje basado en equipos (TBL), estrategia instruccional.

\section{ABSTRACT}

This article describes the steps of the instructional strategy Team-Based Learning or TBL, and a general view of the results of its application in the classroom. Evidence shows that TBL is a practical, economical and effective instructional strategy that generates active learning in teams of students, increases student motivation, facilitates the achievement of student's learning outcomes, and tends to increase academic indicators that impact in the accreditation of careers.

Key words: active and collaborative learning, team-based learning (TBL), instructional strategy. 


\section{INTRODUCCIÓN}

El Aprendizaje Basado en Equipos o TBL (del Inglés: “Team-Based Learning”) es una estrategia instruccional constructivista (Hrynchak y Batty, 2012), que se fundamenta en la teoría de aprendizaje experiencial de Kolb (Roberson y Franchini, 2014), y cuya estructura de actividades en equipos, optimiza la inversión del aula (Wallace et al., 2014), y orienta efectivamente la docencia hacia aprendizaje activo centrado en el estudiante (Fink, 2003).

La estrategia TBL fue desarrollada a principios de 1990 por el Profesor Larry Michaelsen en su curso de Negocios en la Universidad de Oklahoma (USA) en respuesta a 4 hechos claves: 1) aumento del tamaño de su clase (de 40 a 120 estudiantes); 2) su propio descontento con sus clases magistrales; 3 ) el no poder saber qué y cómo pensaban sus estudiantes durante las clases; y 4) el hecho que los estudiantes no tenían oportunidades para resolver, en clases, problemas que sí tendrían que resolver en el mundo real de los negocios. Todo esto lo hizo pensar que mantener una clase tradicional centrada en el profesor y los contenidos sería una pérdida de tiempo (Parmelee et al., 2012).

Así concebida, la estrategia TBL se puede aplicar en clases grandes (hasta 400 estudiantes, pero usualmente 100-150 estudiantes) pero también se puede adaptar a clases pequeñas (menos de 25 estudiantes). TBL requiere 1 solo profesor para facilitar los equipos permanentes de 5-7 estudiantes que resuelven juntos los problemas, casos y/o proyectos, en la misma sala de clases. Por esto, TBL combina el aprendizaje activo y experiencial en grupos grandes y pequeños al permitir que muchos grupos pequeños (los equipos) trabajen juntos en una sala de clases (Michaelsen, Sweet y Parmelee, 2008; Parmelee et al., 2012). Esto parece ser parte del creciente atractivo para usar TBL en la educación superior, ya que TBL incluye una eficiente combinación de recursos (al requerir solo un profesor para facilitar múltiples grupos simultáneamente) junto con altos niveles de participación activa de los estudiantes y aprendizaje significativos, lo cual usualmente se logra al usar estrategias instruccionales con grupos pequeños, separados en diferentes salas y con 1 tutor por grupo (Haidet y Fecile, 2006; Vasan, DeFouw y Compton, 2011). Otros factores que pueden hacer atractivo el uso de TBL son su carácter práctico que no demanda experticia pedagógica de los profesores y que su implementación no depende de cambios de infraestructura o adquisición de equipamiento tecnológico.

En la actualidad, un creciente número de escuelas profesionales universitarias en América, Europa, Asia, Oceanía y África han adoptado la estrategia TBL en cursos de pregrado y postgrado (Haidet, Kubitz y McCormack, 2014; Michaelsen, Knight y Fink 2002; Michaelsen et al., 2008; Nyindo et al., 2014; Parmelee et al., 2012). Los resultados publicados hasta la fecha apoyan la noción respecto a que cuando un curso se implementa con la estrategia TBL, los resultados académicos son equivalentes o mejores que usando formatos de docencia tradicional u otros modelos basados en grupos pequeños (Koles et al., 2005, 2010; Levine et al., 2004; McKiernan, 2003; Michaelsen y Sweet, 2011; Nyindo et al., 2014; Shellenberger et al., 2009; Thomas y Bowen, 2011; Zgheib, Simaan y Sabra, 2010).

La estrategia TBL aplicada a un curso requiere ordenar los contenidos y aprendizajes en uno o más ciclos (usualmente 5-7 ciclos) y cada ciclo se desarrolla en 2 fases, el Proceso de Aseguramiento del Aprendizaje Inicial (fase 1) y las Actividades de Aplicación (fase 2) (Michaelsen et al., 2008). Ambas fases de cada ciclo TBL están caracterizadas por alta retroalimentación de los aprendizajes a través de diversas experiencias de aprendizaje, 
individuales y en equipo, orientadas a exponer y mejorar las habilidades de los equipos de estudiantes para aplicar los contenidos del curso. El objetivo primario de aprendizaje en cursos TBL es ir más allá de solo pasar la materia o los contenidos, asegurando que los equipos de estudiantes tengan la oportunidad de resolver problemas del mundo real usando esos contenidos, teniendo a la vista diferentes opciones de solución y conociendo inmediatamente cual es la mejor opción de solución. El diseño efectivo de problemas, casos y/o proyectos para los equipos, y su adecuada conducción son esenciales para el éxito de la estrategia TBL y la generación de equipos de aprendizajes auto-gestionados (Roberson y Franchini, 2014).

TBL se sustenta en 4 principios fundamentales (Michaelsen et al., 2002):

1) Equipos: Los equipos de estudiantes (5-7 integrantes) son formados por el profesor, distribuyendo lo más homogéneamente todos los activos del curso. Los equipos necesitan ser permanentes para optimizar las opciones de cohesión y evolución como equipos de aprendizaje auto-gestionados.

2) Fase 1: Los equipos son direccionados y preparados durante el Proceso de aseguramiento del aprendizaje inicial (fase 1), para lograr éxito subsecuente en las Actividades de Aplicación (fase 2).

3) Fase 2: Los equipos de estudiantes pasan la mayor parte del tiempo presencial, poniendo a prueba sus conocimientos en las Actividades de Aplicación (fase 2). Los equipos van aprendiendo y cohesionándose para resolver problemas auténticos, interesantes y del mundo real, aplicando en la práctica los conceptos e ideas del curso. De esta manera, los estudiantes generan decisiones complejas, hacen reportes públicos y reciben retroalimentación de calidad e inmediata. Posteriormente, en las discusiones inter-equipos, se propicia un espacio para deliberar y profundizar en los aprendizajes logrados.

4) Responsabilidad: Los estudiantes son motivados a ser responsables de su preparación a través de lecturas previas y pruebas individuales al inicio de la clase, y para contribuir posteriormente al éxito del equipo en las pruebas de equipos, las apelaciones y las actividades de aplicación. La evaluación entre pares (coevaluación) resulta ser un elemento esencial para motivar la responsabilidad y participación de los estudiantes en los equipos.

\section{SECUENCIA DE UN CICLO TBL}

Un curso semestral implementado bajo la estrategia TBL, puede incluir típicamente 5-7 ciclos TBL (Michaelsen et al., 2008). En cada ciclo, la secuencia estructurada de actividades TBL incluye dos fases secuenciales: 1) el proceso de aseguramiento del aprendizaje inicial (fase 1), centrado en la adquisición y construcción de los nuevos contenidos e ideas por parte de los estudiantes, quedando preparados para resolver problemas reales; y 2) las actividades de aplicación (fase 2), que corresponden a la resolución de diversos problemas relevantes, prácticos y significativos del mundo real, por los equipos de estudiantes, en un formato de opciones de soluciones ya preparadas, todos los equipos trabajando en el mismo problema en el aula y con reporte simultáneo de solución por todos los equipos (Michaelsen et al., 2008). 
Un esquema de las dos fases de un ciclo TBL se muestra en la Figura 1. Ambas fases, son necesarias para lograr la transformación del aula, potenciar el aprendizaje en formato de equipos, colocar la adquisición de contenidos fuera del aula y usar la mayor parte del tiempo de clases para el aprendizaje activo con frecuentes pruebas del conocimiento y resolución de problemas relevantes por los equipos con retroalimentación inmediata y evaluación educativa (Fink, 2003).

Figura 1. Las dos fases de un ciclo TBL

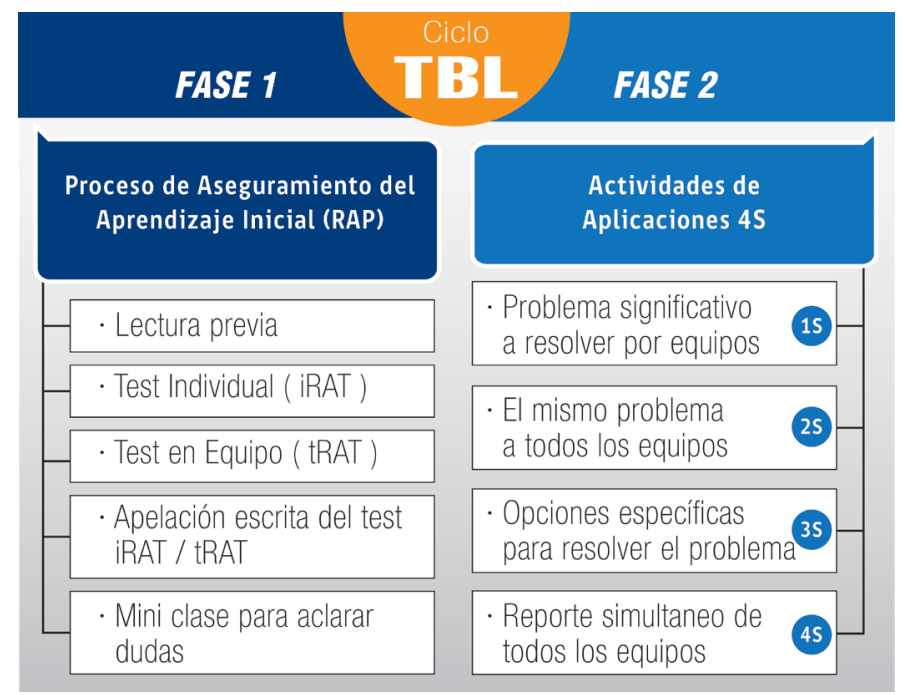

Cada ciclo TBL se inicia con el Proceso de Aseguramiento del Aprendizaje Inicial o fase 1 , que se intenciona a través de 5 actividades secuenciales y progresivas de aprendizaje, como sigue:

1) Lectura previa: Los estudiantes realizan, en forma individual, lecturas previas, seleccionadas o preparadas por el profesor, con la finalidad de llegar preparados a clases. Esto ocurre fuera del aula y al inicio de cada ciclo. Las lecturas pueden incluir capítulos de libros, papers, reportes o monografías, y se pueden complementar con material audiovisual.

2) Test Individual (iRAT): Al llegar al aula, los estudiantes contestan individualmente un test o iRAT (del inglés "Individual Readiness Assurance Test"), que mide la profundidad del estudio y manejo inicial de la lectura previa.

3) Test en Equipo (tRAT): Luego del iRAT, los estudiantes son agrupados por el profesor en equipos para contestar colaborativamente la misma prueba o tRAT (del inglés "Group Readiness Assurance Test"). Esto permite enriquecer sus análisis iniciales y tener retroalimentación inmediata de sus aprendizajes. La retroalimentación inmediata de las respuestas correctas puede ocurrir con el uso de 
tarjetas IF-AT de retroalimentación inmediata (del inglés: "Immediate Feedback Assessment Technique"), durante el mismo trabajo en equipos, ya que las tarjetas IF-AT son tipo "raspe", contienen la pauta de respuestas correctas del tRAT que se indican con un asterisco $(*)$. El uso de tarjetas IF-AT facilita tanto la progresión de análisis en cada equipo, como la asignación de puntajes parciales si el equipo no acertó a la primera elección (Epstein et al., 2002) ${ }^{1}$.

4) Apelación escrita del test iRAT/tRAT: Al finalizar la retroalimentación de los test tRAT y en casos de persistir controversias o dudas sobre una o más preguntas, los equipos de estudiantes escriben y fundamentan con libro abierto sus apelaciones.

5) Mini-Clase: Finalmente, se completa el proceso de la fase 1 con una mini-clase, dirigida por el profesor, con el fin de aclarar todas las dudas de las apelaciones y lograr ordenar y jerarquizar todas las ideas, conceptos y contenidos básicos del módulo (Michaelsen et al., 2002; Parmelee et al., 2012).

A continuación, cada ciclo TBL incluye diversas Actividades de Aplicación 4S, que usualmente constituyen la mayor fracción de tiempo en cada ciclo TBL y permiten gestionar, aplicar y hacer visibles los nuevos aprendizajes de los estudiantes a través de acciones concretas en el aula (Roberson y Franchini, 2014). Ver Figura 2.

Las Actividades de Aplicación $4 S$ corresponden a un formato TBL de gestión del trabajo de los equipos en aula usando: a) problemas significativos y relevantes de la disciplina ("Significative Problem"); b) el trabajo simultáneo de todos los equipos en el mismo problema ("Same Problem"); c) la elección específica de una de las opciones que resuelve el problema ("Specific Choice”); y d) el reporte simultáneo de todos los equipos (“Simultaneous Report”) .

1) Problema significativo: Los equipos trabajan en un problema significativo, atractivo, relevante y real. La calidad del problema condiciona en forma clave la efectividad de la actividad de aplicación. Los problemas deben obligar el uso de conceptos del curso y estar relacionados con los resultados de aprendizaje. Para ello, se puede utilizar un diseño retroactivo: primero enfocarse en el resultado de aprendizaje que quiere lograr, elegir luego una lectura orientada a este resultado de aprendizaje, luego alinear el iRAT con estos conceptos y finalmente generar varios problemas complejos pero factibles de ser resueltos por los equipos con los conocimientos alcanzados.

2) Mismo Problema: Todos los equipos trabajan en el mismo problema. Al entregar a todos los equipos el mismo problema se genera una atmósfera muy proactiva, que crea oportunidades de reflexión, defensa y discusión de ideas y formas de enfrentar y resolver un problema. Se facilita la comparabilidad y la discusión entre los equipos. Cuando se realiza trabajo en equipos en problemas distintos con reporte secuencial, se genera una atmósfera de baja energía, donde los estudiantes no están necesariamente motivados para examinar el proceso de pensamiento y decisiones presentadas por los otros equipos. 
Figura 2. Relación entre el Proceso de Aseguramiento del Aprendizaje inicial (fase 1) y las Actividades de Aplicación 4S (fase 2) de un ciclo TBL

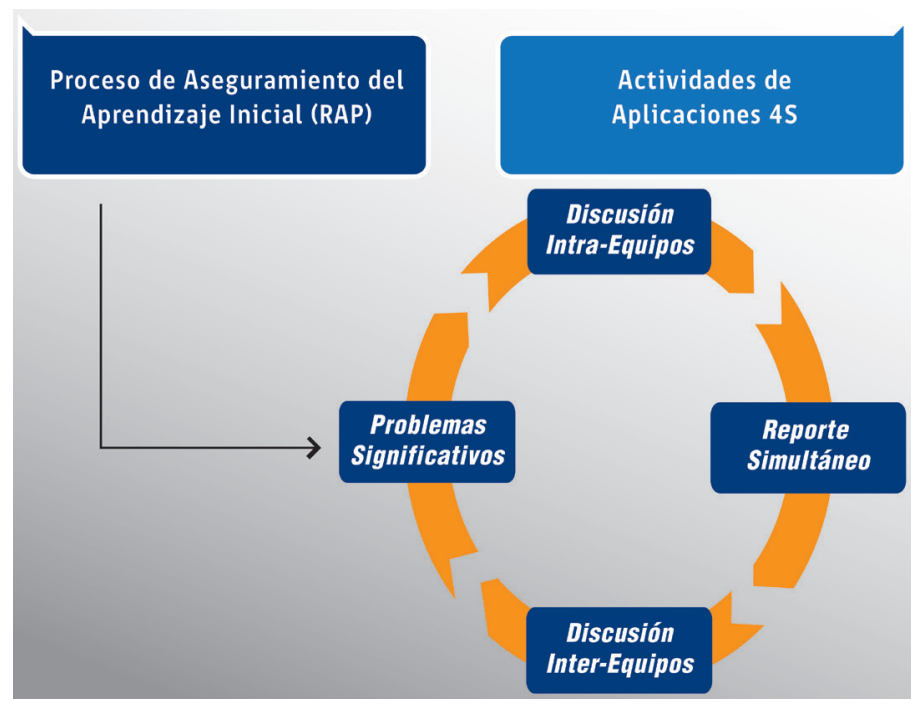

3) Elección específica: Los equipos deben tomar una decisión específica para resolver el problema planteado. El uso de preguntas abiertas ha sido el sustento de grandes esfuerzos docentes para estimular pensamiento crítico en los estudiantes. No obstante, el uso de preguntas abiertas y complejas podría ser un desafío demasiado grande para un novato en el tema de estudio. El aspecto más cuestionable del uso de preguntas abiertas es su ineficiencia al momento de dejar que los alumnos reporten sus respuestas y la dificultad para comparar sus respuestas con los pares. La oportunidad para comparabilidad de decisiones es una de las mayores fortalezas del proceso de reporte del TBL.

4) Reporte simultáneo: Los equipos reportan en forma simultánea sus decisiones. Un reporte simultáneo se logra simplemente levantando una carta que indica una elección específica. Cuando un equipo particular observa que otro equipo ha tomado una decisión diferente, naturalmente ocurre una comparación de racionalidad y de toma de decisiones. Ocurre conversación entre equipos, incluyendo defensa de su propia postura y desafío hacia la decisión del otro equipo. Este reporte requiere que los equipos articulen sus pensamientos hacia los otros equipos, colocando sus pensamientos en palabras. Esto ayuda a la construcción de aprendizajes profundos y significativos. La retroalimentación de pares es inmediata y está enfocada en “¿Cómo llegaste a esa decisión?” antes que “¿Cuál es la respuesta correcta?”.

\section{RESULTADOS DE LA APLICACIÓN DE TBL EN CURSOS UNIVERSITARIOS}

La estrategia TBL ha sido aplicada en forma creciente durante los últimos 25 años en numerosos cursos de enseñanza media, pregrado y postgrado universitario y en otros 
formatos de capacitación (Haidet et al., 2014). Los resultados de la aplicación de TBL en cursos universitarios han sido publicados en revistas, libros, seminarios y congresos ${ }^{2}$.

Diversos reportes apoyan la noción que TBL es una estrategia instruccional efectiva que mejora indicadores académicos y de motivación estudiantil, incluyendo: a) los estudiantes de cursos TBL, tienen un mayor nivel de compromiso (Chung et al., 2009; Clark et al., 2008; Kelly et al., 2005; Levine et al., 2004) o están más contentos y comprometidos con sus aprendizajes (Andersen et al., 2011; Dana, 2007; Letassy et al., 2008; Nicoll-Senft, 2009); b) si en un curso TBL se compara el rendimiento de los equipos de estudiantes con los rendimientos individuales, los peores equipos normalmente sobrepasan a los mejores estudiantes. Los resultados de Larry Michaelsen (Michaelsen, Watson y Black, 1989), después de años de trabajo TBL, indican que el $99.95 \%$ de los equipos supera a su mejor miembro por un promedio de $14 \%$; c) basado en resultados finales con exámenes estandarizados, los estudiantes TBL tienen mejores resultados que los estudiantes no TBL (Grady, 2011; Johnson et al., 2014; Koles et al., 2005; Koles et al. 2010; Letassy et al., 2008; Persky, 2012; Thomas y Bowen, 2011; Zingone et al., 2011); y d) para los estudiantes, estar en una clase grande es considerado un activo beneficioso para el aprendizaje, cuando ocurre bajo el formato TBL (Michaelsen et al., 2002).

Se ha fundamentado que cuando TBL es apropiadamente implementado incluye muchos, sino todos los elementos comunes de las mejores prácticas pedagógicas basadas en la evidencia (Michaelsen y Sweet, 2011), afirmación coherente con su gran alineamiento con los primeros principios de instrucción de Merril (2002) ya que TBL logra promover el aprendizaje al: 1) involucrar a los estudiantes en la resolución de problemas del mundo real; 2) generar activación del conocimiento previo para sustentar y fundamentar el nuevo conocimiento; 3) ofrecer actividades de aplicación en el aula con problemas relevantes y significativos con varias opciones de solución, una de las cuales es la más correcta; 4) obligar a los estudiantes a tomar decisiones tanto en la fase de aseguramiento del aprendizaje inicial como en las actividades de aplicación para aplicar sus conocimientos; y 5) familiarizar y aterrizar las problemáticas disciplinares en las actividades de aplicación para lograr la integración con el mundo del estudiante. Otro apoyo indirecto, pero contundente, proviene de un reciente meta-análisis donde la evidencia validada empíricamente por 225 estudios en cursos de ciencias básicas e ingenierías, concluye que el aprendizaje activo genera mejores resultados en calificación y tasa de aprobación que las estrategias instruccionales centradas en el profesor (Freeman et al., 2014).

Aplicaciones de la estrategia TBL en cursos de pregrado de carreras de Salud y Ciencias Básicas se han desarrollado en diversas universidades chilenas desde el 2010, con buenos resultados en indicadores de tasas de aprobación o, al menos, mayor nivel de satisfacción estudiantil por la docencia en formato TBL. El primer curso TBL en Chile fue el de fisiología médica en la Universidad Católica del Norte, siguiendo con aplicaciones en la Pontificia Universidad Católica de Chile, en la Universidad Viña del Mar y Universidad Iberoamericana (Moraga et al., Manuscrito en preparación) y en la Universidad Pedro de Valdivia (Delgado et al., 2014). Los resultados globales de los estudios piloto en Chile son claros y coincidentes con otros estudios realizados internacionalmente, y permiten confirmar la ventaja comparativa de usar estrategias activas y participativas (Freeman et al., 2014) incluyendo al TBL por sobre la docencia tradicional socrática.

Revisar en http://www.teambasedlearning.org/) 


\section{CONCLUSIONES}

Las razones del éxito relativo y creciente del TBL como estrategia instruccional incluyen su alineamiento con los principios constructivistas del aprendizaje, sus bases neurocientíficas y psicológicas, su razón efectividad costo, la naturaleza práctica de aplicación, su adaptabilidad a las condiciones tradicionales existentes de docencia, sin exigir modificaciones de infraestructura o mayor recurso humano y su adaptabilidad adhesiva y sinérgica con otras metodologías de aprendizaje activo y participativo existentes, tales como aprendizaje por indagación, proyecto, problemas, casos y otros (Dolmans et al., 2014; Wallace et al., 2014).

Diversos estudios apoyan la estrategia de retroalimentación, uso frecuente de test y las opciones de ensayo y error usada durante las 2 fases de los ciclos TBL. En primer lugar las investigaciones en neurociencia cognitiva (Zull, 2002) confirman la teoría de aprendizaje experiencial de Kolb (Roberson y Franchini, 2014), demostrando que nuestro cerebro cambia cuando aprendemos y que las redes neuronales se van consolidando cuando generamos acciones y tenemos experiencias donde es posible cometer errores y aprender de ellos, gracias a retroalimentación inmediata y las emociones (Dragansky et al., 2004). Los errores son considerados ahora como aliados del aprendizaje, como se indica:

en vez de tratar los errores de los estudiantes como obstáculos para su aprendizaje, empecé a darles la bienvenida. Se convirtieron en mis materias primas para ayudar a los estudiantes a construir conocimiento. En lugar de pensar que mi trabajo consistía en erradicar los errores, comencé a estimularlos (Zull, 2004).

Por otro lado, Bransford (2000) enfatiza la diferencia esencial entre comprender y memorizar, citando numerosos estudios que muestran que una simple ruta de aprendizaje no genera transferencia de conocimiento. El psicólogo Cognitivo Willingham (2009), argumenta que "La Memoria es el residuo del pensamiento" (p. 54), implicando que los pensamientos se hacen manifiestos a través de las acciones, y que solo actuando sobre la información, esta se puede transferir desde la memoria de trabajo hacia la memoria de largo plazo. El trabajo de Zull (2002, 2004 y 2011) refuerza estos hallazgos, mostrando que el aprendizaje que no se pone en servicio de una acción, tiende a "dormir" y a través del poco uso se convierte en menos rescatable desde las redes neuronales de la memoria.

En segundo lugar, diversos estudios apoyan la realización de frecuentes test de conocimiento, con retroalimentación inmediata como una herramienta de evaluación y aprendizaje (Epstein et al., 2002; Larsen, Butler y Roediger, 2008, 2009) y no meramente con una instancia de calificación.

Un tema central del TBL es que el aprendizaje de los estudiantes es impulsado por retroalimentación frecuente $y$, cuando es posible, inmediata. Para que esto ocurra, el aprendizaje de los estudiantes y las consecuencias del uso de ese aprendizaje en su pensamiento tiene que ser visible para ellos mismos y para el instructor. Los estudiantes, por lo tanto, son requeridos a actuar frecuentemente de una manera que generen consecuencias que provoquen reflexión y demuestren la visibilidad de sus pensamientos. Mientras más enfocada y concreta sea la acción, más visible será su pensamiento y su aprendizaje, y más inmediatamente útil será la retroalimentación (Roberson y Franchini, 2014).

A fines del siglo XX, el Aprendizaje Basado en Problemas (ABP) se estableció como estrategia de aprendizaje activo líder en las innovaciones educativas en todo el mundo. No obstante, los altos costos de implementación de ABP debido al alto número de tutores, 
junto con la necesidad de infraestructura acorde y de experticia metodológica de los tutores ABP, han comenzado a presionar la migración desde ABP hacia TBL, en diversas carreras y facultades en todo el mundo. De acuerdo a la información disponible en el sitio web oficial de TBL (http://www.teambasedlearning.org/), solo en USA, más de 100 Escuelas de Ciencias de la Salud, al 2014, han dejado atrás el uso de ABP y han iniciado el uso parcial o total de TBL como su estrategia instruccional. Lo mismo ha ocurrido en algunas Escuelas de Ciencias de la Salud en países de Sudamérica como Chile y Perú (Comunicación personal) y algunas escuelas de derecho (Antony Font, comunicación personal). Se puede afirmar que en las instituciones donde se ha desarrollado ABP hay interés por migrar desde ABP a TBL, o al menos existe el interés de combinar lo mejor de ambas estrategias (Dolmans et al., 2014).

En la actualidad, el mayor locus de implementación del TBL en el mundo está en las Escuelas de Medicina, siendo un ejemplo interesante el mejor rendimiento de los estudiantes de la Universidad de DUKE, en Singapur, que usa estrategia TBL, por sobre los resultados de los estudiantes de la Escuela de Medicina de la misma universidad en USA que utiliza docencia centrada en el profesor y en los contenidos.

El cúmulo de evidencia publicada indica que TBL presenta una alta y atractiva razón efectividad costo debido a los altos resultados de efectividad académica y motivación estudiantil y bajos costos de implementación (Vasan et al., 2011). Esto posiciona al TBL como una estrategia de elección para generar aprendizaje activo y colaborativo, sin mayores cargas pecuniarias para las instituciones. Otro aspecto beneficioso del TBL es su carácter práctico y estructurado, que facilita su implementación paso a paso, sin necesidad de capacitación exhaustiva. Un facilitador TBL que es experto en su disciplina no debe ser experto en la estrategia TBL, situación opuesta a la de Aprendizaje Basado en Problemas (ABP), en la que el tutor puede incluso no ser experto en la disciplina, pero tiene que ser experto en la estrategia ABP para lograr conducir apropiadamente el trabajo colaborativo en grupos pequeños de estudiantes (Hmelo-Silver, 2004).

\section{AGRADECIMIENTOS}

DM y JS agradecen a Daniel Moraga Azema (QEPD), por la confección de las dos figuras de este artículo.

\section{REFERENCIAS BIBLIOGRÁFICAS}

Andersen, E., Strumpel, C., Irene Fensom, I., \& Andrews, W. (2011). Implementing Team Based Learning in Large Classes: Nurse Educators' Experiences. International Journal of Nursing Education Scholarship, 8(1), 1-16.

Bransford, J. D., Brown, A. L., Cocking, R. R., Donovan, M. S., \& Pellegrino, J. W. (2000). How people learn: Brain, mind, experience, and school (expanded ed.). Washington, DC: National Academy Press.

Chung, E., Rhee, J., Baik, Y., \& A, O. (2009). The effect of team-based learning in medical ethics education. Med Teach, 31, 1013-1017.

Clark, M., Nguyen, H., Bray, C., \& Levine R. (2008). Team-based learning in an undergraduate nursing course. Nursing Education, 47(3), 111-117. 
Dana, S. (2007). Implementing Team-Based Learning in an Introduction to Law Course. Journal of Legal Studies Education, 24(1), 59-108.

Delgado, M., Fasce, E., Pérez, C., \& Rivera, N. (2014). Cambios en el aprendizaje autodirigido asociados a la implementación de una metodología de aprendizaje basado en equipo (TeamBased Learning). Rev Educ Cienc Salud, 11(2), 161-165.

Dolmans, D., Michaelsen, L., van Merrienboer, J., \& van der Vleuten, C. (2014). Should we choose between problem-based Learning and team-based learning? No, combine the best of both worlds! Medical Teacher, 37(4), 1-6.

Draganski, B., Gaser, C., Busch, V., Schuierer, G., Bogdahn, U., \& May, A. (2004). Neuroplasticity: changes in grey matter induced by training. Nature, 427(6972), 311-312.

Epstein, M., Lazarus, A., Calvano, T., Matthews, K., Hendel, R., Epstein, B., \& Brosvic, G. (2002). Immediate Feedback Assessment Technique promotes learning and corrects inaccurate first responses. The Psychological Record, 52, 187-201.

Fink, D. (2003). Creating Significant Learning Experiences: An Integrated Approach to Designing College Courses. San Francisco: Jossey-Bass.

Freeman, S., Eddy, S., McDonough, M., Smith, M., Okoroafor, N., Jordt, H., \& Wenderoth, M. (2014). Active learning increases student performance in science, engineering, and mathematics. Proceedings of the National Academy of Sciences (PNAS), 111(23), 8410-8415.

Grady, S. (2011). Teachers Topics: Team-based learning in pharmacotherapeutics. Am J Pharm Educ, 75(7), 136-142.

Haidet, P., \& Fecile, M. (2006). Team-based learning: A promising strategy to foster active learning in cancer education. Journal of Cancer Education, 21(3), 125-128.

Haidet, P., Kubitz, K., \& McCormack, W. (2014). Analysis of the team-based learning literature: TBL comes of age. Journal on Excellence in College Teaching, 25(3-4), 303-333.

Hmelo-Silver, C. (2004). Problem-based learning: What and how do students learn? Educ Psych Review, 16(3), 235-266.

Hrynchak, P., \& Batty, H. (2012). The educational theory basis of team-based learning. Med Teach, 34(10), 796-801.

Johnson, J., Edward Bell, E., Bottenberg, M., Darla Eastman, D., Sarah Grady, S., Koenigsfeld, C., Maki, E., Kristin Meyer, K., Phillips, C., \& Schirmer, L. (2014). A Multiyear Analysis of TeamBased Learning in a Pharmacotherapeutics Course. Am J Pharm Educ, 78(7), 142-150.

Kelly, P., Haidet, P., Schneider, V., Searle, N., Seidel, C., \& Richards, B. (2005). A Comparison of InClass Learner Engagement Across Lecture, Problem-Based Learning, and Team Learning Using the STROBE Classroom Observation Tool. Teaching and Learning in Medicine, 17(2), 112-118.

Koles, P., Nelson, S., Stolfi, A., Pamelee, D., \& DeStephen, D. (2005). Active learning in a year 2 pathology curriculum. Med Educ, 39(10), 1045-1055.

Koles, P., Stolfi, A., Borges, N., Nelson, S., \& Parmelee, D. (2010). The impact of team-based learning on medical students' academic performance. Acad Med, 85(11), 1739-1745.

Larsen, D., Butler, A., \& Roediger, H. (2008). Test enhanced learning in medical education. Medical Education, 42(10), 959-966.

Larsen, D., Butler, A., \& Roediger, H. (2009). Repeated testing improves long term retention relative to repeated study: a randomised controlled trial. Medical Education, 43(12), 1174-1181.

Letassy, N., Fugate, S., Medina, M., Stroup, J., \& Britton, M. (2008). Using team-based learning in an endocrine module taught across two campuses. Am J Pharm Educ,72(5). Recuperado desde: http://www.ncbi.nlm.nih.gov/pmc/articles/PMC2630128/

Levine, R., O’Boyle, M., Haidet, P., Lynn, D., Stone, M., Wolf, D., \& Managua, F. (2004). Transforming a clinical clerkship with team learning. Teach Learn Med, 16(3), 270-275.

McKiernan, M. (2003). Team-based learning enhances long-term retention and critical thinking in an undergraduate microbial physiology course. Microbiol Educ J, 4(1), 3-12.

Merril, D. (2002). First principles of instruction. EE.UU.: John Wiley and Sons. 
Michaelsen, L., Knight, A., \& Fink, D. (2002). Team-Based Learning: A Transformative Use of Small Groups for Large and Small Classes. Westport, Conn: Bergin \& Garvey.

Michaelsen, L., \& Sweet, M. (2011). Team-based Learning. New Directions for Teaching and Learning, 128, 41-51.

Michaelsen, L., Sweet, M., \& Parmelee, D. (2008). Team-based learning: Small-group learning's next big step. New Directions for Teaching and Learning, 116, 1-104.

Michaelsen, L. K., Watson, W. E., \& Black, R. H. (1989). A realistic test of individual versus group consensus decision making. Journal of Applied Psychology, 74(5), 834-839.

Moraga, D., Soto, Y., Malatesta, D., \& Olivares, C. Efecto de la aplicación de TBL en ciencias básicas. Manuscrito en preparación.

Nicoll-Senft, J. (2009). Assessing the impact of team-based learning. Journal on Excellence in College Teaching, 20, 27-42.

Nyindo, M., Kitau, J. E., Kapanda, G., Matowo, J., Francis, P., \& Bartlett, J. (2014). Introduction of Team-Based Learning at Kilimajaro Christian Medical University College: Experience with the Ectoparasites module. Medical Teacher, 36(4), 308-313.

Parmelee, D., Michaelsen L., Cook, S. \& Hudes, P. (2012). Team-based learning: A practical guide: AMEE Guide No. 65. Medical Teacher, 34, e275-e287.

Persky, A. (2012). The Impact of Team-Based Learning on a Foundational Pharmacokinetics Course. American Journal of Pharmaceutical Education, 76(2). doi:10.5688/ajpe76231

Roberson, B., \& Franchini, B. (2014). Effective task design for the TBL classroom. Journal on Excellence in College Teaching, 25(3-4), 275-302.

Shellenberger, S., Seale, J., Harris, D., Johnson, J., Dodrill, C., \& Velasquez, M. (2009). Applying team-based learning in primary care residency programs to increase patient alcohol screenings and brief interventions. Acad Med, 84(3), 340-346.

Thomas, P., \& Bowen, C. (2011). A controlled trial of team-based learning in an ambulatory medicine clerkship for medical students. Teach Learn Med, 23(1), 31-36.

Vasan, N., DeFouw, D., \& Compton, S. (2011). Team-based learning in anatomy: an efficient, effective, and economical strategy. Anatomical Sciences Education, 4(6), 333-339.

Wallace, M., Walker, J., Braseby, A., \& Sweet, M. (2014). “Now, what happens during class?” Using team-based learning to optimize the role of expertise within the flipped classroom. Journal on Excellence in College Teaching, 25(3-4), 253-273.

Willingham, D. T. (2009). Why don't students like school? San Francisco, CA: Jossey-Bass.

Zgheib, N., Simaan, J., \& Sabra, R. (2010). Using team-based learning to teach pharmacology to second year medical students improves student performance. Med Teach, 32(2), 130-135.

Zingone, M., Franks, A., Guirguis, A., George, C., Howard-Thompson, A., \& Heidel, R. (2010). Comparing team-based and mixed active-learning methods in an ambulatory care elective course. American Journal of Pharmaceutical Education, 74, 1-7.

Zull, J. (2002). The art of changing the brain. Sterling, VA:Stylus.

Zull, J. (2004). The art of changing the brain. Teaching for Meaning, 62(1), 68-72.

Zull, J. (2011). From brain to mind. Sterling, VA: Stylus. 
